\title{
Cortical activation during a cognitive challenge in patients with chronic temporal lobe epilepsy-a dynamic SPECT study
}

\author{
P.J. Kirkpatrick ${ }^{3}$, R. Morris ${ }^{1}$, G.M.S. Syed ${ }^{2}$ and C.E. Polkey ${ }^{1}$ \\ 'Departments of Neurosurgery and Psychology, Maudsley Hospital, DeCrespigny Park, \\ Camberwell, London SE5 8AZ, ${ }^{2}$ Department of Nuclear Medicine, King's College Hospital, \\ Denmark Hill, London SE5 9RS and ${ }^{3}$ University Department of Neurosurgery, Block A, \\ Level 4, Addenbrookes Hospital, Cambridge CB2 2QQ, UK
}

Correspondence to: P.J. Kirkpatrick at above address

In a pilot group of six patients suffering from chronic temporal lobe epilepsy, single photon emission computerized tomography (SPECT) has been used to image the changes in relative cerebral blood flow between rest (static scan) and conditions of cognitive activation (activated scan). The cognitive challenge used during activation comprised a test of word memory, and the performance was expressed as a word memory score (WMS) for each individual. An activation index (AI) was calculated from the mean normalized density counts in specific regions of interest (ROIs), and values obtained were analysed for correlation with the WMS. The mean AI was increased significantly in the right lateral temporal cortex, the right and left inferior frontal regions, the left temporal pole, and the right medial temporal cortex. A positive correlation with the WMS was found in the medial temporal cortices, and this relationship was significant for the right medial temporal ROI.

Keywords: Cognition - Memory - SPECT - Temporal lobe epilepsy

\section{INTRODUCTION}

Lateralization of cognitive function is an essential part of assessing patients with temporal lobe epilepsy (TLE) who are being considered for resective surgery. Removal of cortical regions important for memory will result in an amnesic syndrome (Scoville and Milner, 1957; Milner, 1975). Present-day methods for establishing lateralization of memory largely depend on detailed neuropsychological investigations (Jones Gotmans, 1987) including the invasive carotid amytal (Wada) test (Wada, 1949; Milner et al., 1962; Wada et al., 1975; Rausch and Langfitt, 1990). These techniques are time consuming and unreliable. For example, in the Wada test it is not known precisely which parts of the brain are anaesthetized during the administration of intracarotid sodium amytal, and there is evidence that the barbiturate may affect each hemisphere differently (Biersack et al., 1987; Ryding et al., 1988). Consequently, the data obtained can be difficult to interpret and the results are often equivocal (Powell et al., 1987).

A more objective method of establishing cortical regions subserving higher cognitive function is needed. In this regard, techniques that can image alterations in regional cerebral blood flow ( $\mathrm{rCBF}$ ) may be useful as they have been shown to reflect changes in regional brain activity (Risberg, 1980; Mathew et al., 1982). In epileptics such methods have already helped in localizing the onset of seizures (Kuhl et al., 1980; Bonte et al., 1983; Lee et al., 1988; Engel, 1988; Ryding et al., 1989; Rowe et al., 1989), and have been used to correlate the psychological deficits of such patients with regional cerebral hypoperfusion (Homan et al., 1989). In other patients they can show specific changes in regional cerebral perfusion during different types of hallucinations (Musalek et al., 1989). To date these techniques have not been used in an attempt to lateralize cognition in epileptics. However, encouraged by earlier results in the use of cognitively activated single photon emission computerized tomography (SPECT) imaging in patients who have suffered subarachnoid haemorrhage (Kirkpatrick et al., 1991), we have used a similar method to acquire rest and cognitively activated SPECT scans in six patients who suffer from chronic TLE.

We have attempted to measure the response of $\mathrm{rCBF}$ to a cognitive challenge that assesses word memory. This psychological variable is relatively simple to measure, and is an important component of the psychometric assessment and Wada test as used routinely in this unit (Powell et al., 1987). Lateralization of word memory is also of considerable importance when contemplating resective 
TABLE I. Location of onset of seizure electrical activity established using surface EEG recordings and telemetry with foramen ovale (FO) electrodes, and comparison with side of relative temporal lobe hypoperfusion on the static SPECT images

\begin{tabular}{lccc}
\hline Patient & Surface EEG & FO telemetry & $\begin{array}{c}\text { Static SPECT image } \\
\text { (relative temporal } \\
\text { hypoperfusion) }\end{array}$ \\
\hline 1 & L subfrontal & L medial temporal & $\mathrm{L}$ \\
2 & L temporal & L medial temporal & $\mathrm{L}$ \\
3 & $\mathrm{R}$ sphenoidal & R medial temporal & None \\
$4^{1}$ & L middle temporal & Bilateral medial temporal & $\mathrm{R}$ \\
5 & R anterior temporal & R medial temporal & $\mathrm{R}$ \\
$6^{1}$ & Bilateral sphenoidal & Bilateral medial temporal & $\mathrm{R}$ \\
\hline${ }^{1}$ Patients later shown to have a right medial temporal epileptic focal during depth electrode investigations.
\end{tabular}

neurosurgery. Our provisional results suggest that cognitive activation of specific regions of the cerebral cortex can be demonstrated with SPECT in epileptic patients.

\section{METHODS}

\section{Clinical material}

This pilot study had full approval from the Maudsley Ethical Committee. Informed consent was obtained from all participants.

Six patients awaiting investigation for chronic temporal lobe epilepsy (complex partial seizures, mean duration 22.1 years) were randomly selected from the waiting list. All had been referred to the unit for standard assessment of their suitability for temporal lobe surgery. This type of patient was chosen because they were already scheduled for higher cognitive assessment including a Wada test. All patients had high resolution head CT scans (GE9800) which included five $0.5 \mathrm{~mm}$ slices orientated along the long axis of the temporal lobe which provided high quality images of the medial temporal regions (Kirkpatrick et al., 1991). The middle temporal slice transected the temporal pole and was used to assess the anatomical alignment of the temporally orientated SPECT scan (see below).

\section{Routine epilepsy investigations}

Each patient began standard investigation for epilepsy. All received scalp EEG and telemetry with foramen ovale (FO) electrodes to localize the onset of seizure activity (Table I). A routine carotid amytal test was carried out to determine speech dominance (Table II). The results of these investigations were not available to those conducting the cognitive challenge during SPECT imaging (see below) and subsequent image analysis.

\section{SPECT imaging}

One static and one activated scan was obtained for each patient within a 2 week period. For each investigation, 500
Mq of [ $\left.{ }^{99} \mathrm{Tc}\right] \mathrm{HM}-\mathrm{PAO}$ (Ceretec, Amersham International) was administered intravenously at a designated time (see below). Scans were acquired on a dedicated multicrystal high resolution transaxial tomography system SME 810 (Strichman Medical Equipment). The system is regularly calibrated for energies, efficiencies, and offsets according to a calibration menu included in the software which then corrects deviation. The system response was linear to varying amounts of standard isotope $(r=0.99)$, and spatial resolution was $7.7 \pm 0.5 \mathrm{~mm}$. Procedures and dosage of isotope were approved by ARSAC.

The patient's head was orientated by using an optical marker to align the orbito-meatal axis. The patient's head was strapped in position to prevent movement during the scan.

\section{Static scan}

Images were acquired with the patient lying quietly and the eyes open for $20 \mathrm{~min}$. Five minutes before intravenous administration of the isotope the patient was shown a series of blank cards which continued for $10 \mathrm{~min}$ after the injection.

\section{Activation scan}

A continuous verbal recognition memory test was used in the activation scan phase of the study. This test involved presenting a series of 240 single words, split into 20 blocks of 12 words. These words were presented visually on cards at a rate of approximately one word per $4 \mathrm{~s}$. Initially the words were all different, but after a set period they began

TABLE II. Word memory scores

\begin{tabular}{ccccccc}
\hline \multicolumn{8}{c}{ Patient } & & \\
\hline 1 & 2 & 3 & 4 & 5 & 6 \\
\hline 53 & 88 & 77 & 36 & 59 & 72 \\
\hline
\end{tabular}


to repeat. For each word the task was to state "yes" or "no" as to whether it had been seen before. The test was designed such that after the first three blocks of cards there was an equal number of new and repeated words overall. Thus in each block of 12 words, six were new words and six had been shown before. Each new word had only one repeat, and this was shown within the next three blocks. The interval between presentation and repeat of a word was arranged in pseudo-random order according to these constraints. The cognitive challenge lasted for approximately $15 \mathrm{~min}$, and the isotope was injected after the 40th card. A word memory score (WMS) was calculated by subtracting the number of false positive responses from the number of correct positive responses (Table II).

\section{Image analysis}

Each SPECT investigation consisted of multiple, $15 \mathrm{~mm}$ thick overlapping scans orientated parallel to the orbitomeatal axis. In addition, three temporal images were obtained with the slice orientation along the temporal lobe axis. Scans were acquired within $75 \mathrm{~min}$ of isotope injection. Reconstruction was carried out using inverse count dependent Weiner filters and iterative reconstruction on a Macintosh IIX computer. Using the scanner's software (SME version 2.66), user-dependent cortical and subcortical regions of interest (ROIs) were defined. A map of the surface of the brain was also drawn for each static image and superimposed on the equivalent trace from the activated scan to ensure that the slice orientation/location had been maintained. Temporal orientated slices allowed medial and lateral temporal cortex to be resolved (Fig. 1). The raw density values obtained from each ROI were normalized by calculating the standard ratio:

$$
\frac{\text { mean ROI activity }}{\text { mean cerebellar activity }}
$$

or

$$
\frac{\text { mean ROI activity }}{\text { mean global cerebral activity }}
$$

The same ROI templates outlined for the static scans were digitally stored and superimposed on the activated images from the same patient using the outline of the brain surface for accurate realignment. Standardized mean densities for activiation ROIs were subtracted from values obtained in corresponding ROIs in the static scan and expressed as an activation index (Mathew et al., 1982):

$$
\text { activation index }(\mathrm{AI})=\frac{\text { activation } \mathrm{rCBF}-\text { static } \mathrm{rCBF}}{\text { activation } \mathrm{rCBF}+\text { static } \mathrm{rCBF}} \times 100 \%
$$

The ROI analysis was carried out by G.M.S.S. whilst blind to the clinical details. Activation ratios were analysed for correlation with the WMS.

\section{RESULTS}

\section{EEG investigations (Table I)}

Two patients showed definite right-sided (Nos 3 and 5), and two showed left-sided (Nos 1 and 2) medial temporal onset of seizure activity. In the remaining two patients (Nos 4 and 6) bilateral medial temporal onset was seen. Subsequent recordings using depth electrodes indicated a right-sided onset of seizure activity in both of these cases.

\section{Carotid amytal (Table III)}

All patients were left hemisphere speech dominant. Word recognition indicated lateralization of memory in only one patient (No. 2) which was right sided. In the remainder there was evidence of bilateral memory function, but these results were equivocal.

\section{Word memory score}

The WMS obtained during acquisition of the activated scans are listed in Table III.

\section{Static scan images}

Visual examination of the static SPECT scans indicated clear asymmetry of temporal lobe perfusion in all patients (Fig. 2). Three showed relative left-sided, and three rightsided temporal regions of hypoperfusion (Table I). Five out of the six patients demonstrated relative temporal lobe hypoperfusion on the side ipsilateral to the epileptic focus (Table I). The medial temporal region was identified in all scans on the same slice number, and the cortical outline corresponded closely with those obtained from the midtemporal slice obtained on the CT scan.

\section{$\mathrm{ROI}$ analysis}

The $99 \%$ confidence interval for the mean AI from all ROIs was -0.753 to $+1.41 \%$ (normalized to the cerebellum). Significantly raised rCBF was seen in both temporal lobes and was maximal in the right lateral temporal cortex (mean $\mathrm{AI}=+3.38 \%$ ), right inferior frontal cortex (mean $\mathrm{AI}=3.18 \%$ ), left inferior frontal cortex (mean $\mathrm{AI}=3.0 \%)$, left temporal pole (mean $\mathrm{AI}=+2.68 \%)$ and right medial temporal cortex (mean $\mathrm{AI}=1.45 \%$ ). No other ROI showed any significant activation. Further analysis of the temporal cortical areas showed a positive correlation between the mean of the activation indices from the left and right medial temporal ROIs and the WMS $\left(R^{2}=84.5 \% ; p=0.0095\right)$. Lateral temporal ROIs showed no such correlation. When the individual medial temporal ROIs were analysed, positive correlation with WMS was most striking for the right side $\left(R^{2}=91.28 \% ; p=0.0029\right)$. This remained significant after a Bonferroni correction at the $p=0.05$ level. These changes in mean AI were not accompanied by a correlated decrease in any temporal or extratemporal ROI. These relationships held true when ROI activity was normalized to the mean global cerebral activity. 


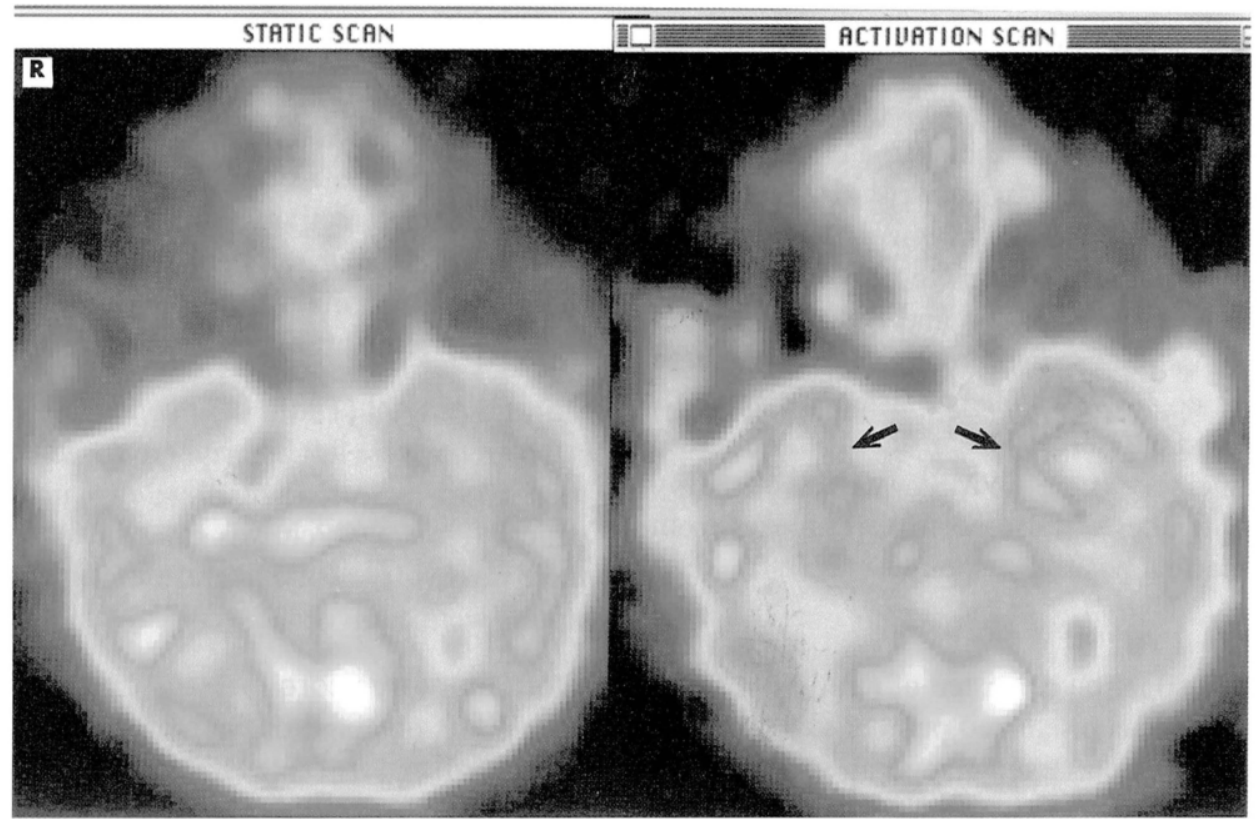

FIG. 1. Static (left) and activated (right) SPECT scans orientated along the long axis of the temporal lobe. A cognitive challenge has activated both medial temporal cortices (arrowed).

\section{DISCUSSION}

The six patients in this study were pre-selected in that they all had suffered from chronic temporal lobe epilepsy, and were awaiting investigations to assess their suitability for epilepsy surgery. They underwent standard investigations including detailed EEG telemetry, and a Wada test. The

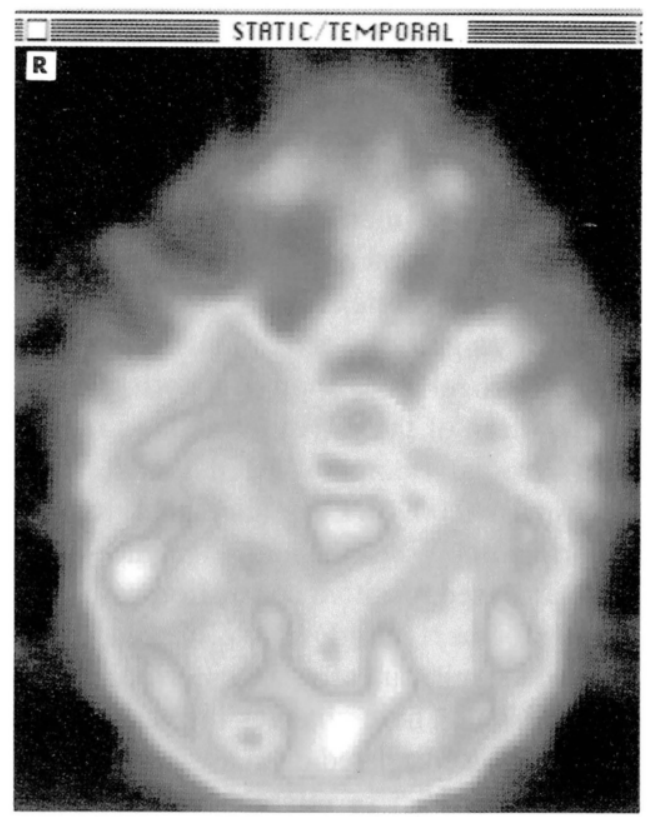

FIG. 2. Static SPECT image taken at a level through the upper temporal lobe. Relative hypoperfusion is seen in the left temporal pole. only additional investigations carried out for the purpose of the study were the two SPECT scans. Although the Wada test indicated left-sided speech dominance in all patients, the test indicated word memory lateralization in only one patient, with the remainder showing equivocal results. The results for this small group of patients serve to demonstrate the difficulties encountered in lateralizing cognition before resective surgery for intractable epilepsy can be contemplated.

\section{Use of SPECT for determination of rCBF}

The use of $\left.{ }^{[99} \mathrm{Tc}\right] \mathrm{HM}-\mathrm{PAO}$ as a sensitive indicator for $\mathrm{rCBF}$ in this study is justified as the relationship between the parenchymal uptake of isotope and blood flow is linear provided the latter does not greatly exceed the normal physiological range (Lassen et al., 1988). Excessively high $\mathrm{CBF}$ was not seen in any patient in the present series. However, relative temporal hypoperfusion was evident on the side of the epileptic focus in five of the six patients. These observations therefore lend some support to the use of static SPECT imaging in localizing epileptic foci in chronic temporal lobe epilepsy (Kuhl et al., 1980; Bonte et al., 1983; Engel, 1988; Lee et al., Ryding et al., 1989; Rowe et al., 1989).

The most important parameters in this type of study are quantification reliability, and the accuracy of the relocation of ROIs from static to activated scan. Quantification regression with standard isotope doses scored highly $(r=0.99)$ so mean counts acquired with the SME system from a specified ROI are accurate. By defining an activation index, the selectivity of the analysis is improved 
at the expense of sensitivity. Thus even small relative changes in the AI are likely to be significant and represent relatively larger changes in mean normalized density counts for individual ROIs between scans (Mathew et al., 1982).

\section{Anatomical resolution}

By using an optical marker to align the head in the scanner gantry, and firm straps to secure the head in position whilst acquiring a scan, we found that the brain outlines from the respective static and activated scans coincide very closely at all scan levels in all patients. Previous experience using the SME SPECT scanner indicated that head movement during image acquisition was easily recognizable by this method (Kirkpatrick et al., 1991). Hence it is likely that any change in the normalized density counts between scans reflects a relative change in $\mathrm{rCBF}$ from comparable ROIs.

The medial temporal ROI was represented on one slice only, and we found our methods reliable in imaging this area on the expected $15 \mathrm{~mm}$ thick slice. The morphology of this slice also compared favourably with the temporally orientated CT scan which is the method adopted in our unit to detect sclerosis of the medial temporal region in epileptics (Kirkpatrick et al., 1993). The anatomical resolution of the SME topography system has been considered in detail elsewhere (Todd-Pokropek, 1984; King et al., 1984; Stoddard, 1987).

\section{Interpretation of cognitive activation}

In a heterogeneous group of epileptic patients, cognitively activated scanning demonstrated a significant change in rCBF in a few specific regions. Activation in the right medial temporal area correlated closely with the level of cognition achieved by the individual patients. With such a small pilot group of patients, adequate controls are missing. Speech activation was not controlled in our methodology as patients remained silent during acquisition of the static scan. Hence we cannot formally resolve the effects of word memory, vision and vocalization. However, we feel that it is unlikely that the vocalization was responsible for right medial temporal activation since all patients spoke ("yes" or "no") the same number of times during the task, and all were proven to be left hemisphere dominant during the Wada test. Significant increases in the mean AI in the interior frontal regions bilaterally and the right lateral cortex were noted which did not correlate with the WMS. It is more likely that increased blood flow to these regions may partly represent functional activation during the motor responses involved in speech as inferior frontal somatotopic representation of the facial musculature occurs bilaterally, and Broca's area usually occupies the left inferior frontal region (Penfield and Rasmussen, 1950).
TABLE III. Speech dominance and word recognition scores from the Wada test

\begin{tabular}{lcccccc}
\hline & \multicolumn{7}{c}{ Patient } \\
\cline { 2 - 7 } & 1 & 2 & 3 & 4 & 5 & 6 \\
\hline SD & $\mathrm{L}$ & $\mathrm{L}$ & $\mathrm{L}$ & $\mathrm{L}$ & $\mathrm{L}$ & $\mathrm{L}$ \\
WORD L & 3 & -1 & 4 & -1 & 1 & 1 \\
WORD R & 1 & 2 & 2 & 1 & 3 & 0 \\
CONTROL & 1 & 4 & 1 & 1 & 3 & 5 \\
\hline
\end{tabular}

SD, speech dominance; WORD L, word recognition score for left hemisphere; WORD R, word recognition score for right hemisphere; CONTROL, word recognition for both hemispheres.

The results of formal psychological assessment (carried out by R.M.) have been omitted since correlation and interpretation of these additional data would be impossible in such a small group. However, it is notable that the WMS did correlate with the mean activation ratios derived from both medial temporal regions, and this may reflect the findings of the Wada test where some word recognition was demonstrated from both hemispheres in three cases (Patients 1, 3 and 5, Table III). The findings are encouraging as the regions of activation were in the medial temporal areas as predicted from historical data (Smith, 1989). Further, a recent larger independent controlled study using positron emission tomography (PET) in normal human volunteers reports selective increases in $\mathrm{rCBF}$ within the right hippocampal region specific to a distinct type of word memory function (Squire et al., 1992). The authors suggest that the right hippocampus is activated because the memory task was driven by a visual form of word. Furthermore, right frontal activation was noted in these PET studies even when the patients remained silent. These findings using cognitively activated PET appear to agree very closely with our own uncontrolled observations using the more widely available SPECT despite the heterogeneity of our patients.

\section{SUMMARY}

Cognitively activated SPECT scanning in a small pilot group of patients with chronic temporal lobe epilepsy has demonstrated a positive correlation between an activation word memory challenge and regional cerebral blood flow to the medial temporal regions. No such association was seen in any other part of the brain analysed. The results of this pilot study are presented as a stimulus to the further investigation of such patients by imaging cognitively activated CBF. Carefully designed controlled studies using large numbers of patients will be necessary to decipher the complex process that are known to affect cortical blood flow in both pathological and non-pathological states. However, the efficiency of our methods can be improved by selective scanning of the temporal and frontal cortex 
and introducing the split dose technique of Shedlack et al. (1991). This allows both static and activated scan acquisitions in quick succession without repositioning of the patient within the scanner gantry, and should not theoretically alter the relative activation index. Since the Wada remains the gold standard in assessing laterality of memory in clinical practice, the involvement of epileptic patients who are planned for resective surgery is to be encouraged. The reduced dose of radioactive isotope required in the split dose technique would allow an additional postoperative SPECT assessment of these patients, thereby providing an opportunity to verify any conclusions as to the laterality of memory drawn from preoperative activation scans.

\section{REFERENCES}

Biersack HJ, Linke D, Brassel F, et al. (1987) Technetium-99m HM-PAO brain SPECT in epileptic patients before and during unilateral hemisphere anaesthesia (Wada test): Report of three cases. Journal of Nuclear Medicine, 28, 1763-1767.

Bonte FJ, Devous MD, Stokely EM and Homan RW (1983) Single photon tomographic determination of regional cerebral blood flow in epilepsy. Archives of Neurology, 40, 267-270.

Engel J (1984) The use of PET scanning in epilepsy. Annals of Neurology, 15 (Suppl.), S180-191.

Homan WR, Paulman RG, Devous MD, et al. (1989) Cognitive function and regional blood flow in partial seizures. Archives of Neurology, 46, 964-970.

Inugami A, Kanno I, Uemura D, et al. (1988) Linearisation correction of Tc-labelled HMPAO image in terms of regional CBF distribution: comparison to $\mathrm{C}^{15} \mathrm{O}_{2}$ inhalation steady state method measured by positron emission tomography. Journal of Cerebral Blood Flow Metabolism, 8, S52-60.

Jones Gotmans M (1987) Commentary; Psychological evaluation-testing hippocampal function. In: Surgical Treatment of the Epilepsies (Ed. J Engel). Raven Press, New York.

King MA, Schwinger RB, Doherty PW, et al. Two dimensional filtering of SPECT images using Metz and Weiner filters. Journal of Nuclear Medicine, 25, 1234-1240.

Kirkpatrick PJ, Shar S, Pickering A, et al. (1991) Cognitive function and cerebral blood flow patterns following subarachnoid haemorrhage. Nuclear Medicine Communications, 12, 280.

Kirkpatrick PJ, Honavar M, Janota I and Polkey CE (1993) Control of temporal lobe epilepsy following en bloc resection of low grade tumours Journal of Neurosurgery, 78, 19-25.

Kuhl DE, Engel J, Phelps ME and Selin C (1980) Epileptic patterns of local cerebral metabolism and perfusion in humans determined by emission computered tomography of ${ }^{17} \mathrm{FDG}$ and ${ }^{13} \mathrm{NH}_{3}$. Annals of Neurology, 8, 348-360.

Lassen NA, Anderson AR, Frieberg L and Paulson OB (1988) The retention of Tc $99 \mathrm{~m} \mathrm{D,1-HMPAO}$ in the human brain after intracarotid bolus injection: a kinetic analysis. Journal of Cerebral Blood Flow Metabolism, 8, S13-22.

Lee BI, Markland ON, Wellman HN, et al. (1988) HIPDMSPECT in patients with medically intractable complex partial seizures. Ictal study. Archives of Neurology, 45, 397-402.

Mathew RJ, Duncan GC, Weinman ML, et al. Regional cerebral blood flow in schizophrenia. Archives of General Psychiatry, 39, 1121-1124.

Milner B (1975) Psychological aspects of focal epilepsy and its management. Advances in Neurology, 8, 299-314.
Milner B, Branch C and Rasmussen T (1962) Study of short term memory after intracarotid injection of sodium amytal. Transactions of the American Neurological Association, 87, 224-226.

Musalek M, Podreka HW, Suess E, et al. (1989) Regional brain function in hallucinations: A study of regional cerebral blood flow with 99m-TC-HMPAO-SPECT in patients with auditory hallucinations, tactile hallucinations and normal controls. Comparative Psychiatry, 30, 99-108.

Penfield W and Rasmussen T (1950) The Cerebral Cortex of Man: A Clinical Study of Localisation of Function. Macmillan, New York.

Powell GE, Polkey CE and Canavan AGM (1987) Lateralising of memory functions in epileptic patients by use of the sodium amytal (Wada) technique. Journal of Neurology, Neurosurgery and Psychiatry, 50, 665-672.

Rausch R and Langfitt JT (1990) Memory estimation during the intracarotid sodium amobarbital procedure: problems and perspectives. In: Surgery of the Epilepsies (Ed. H Luders). Raven Press, New York.

Risberg J (1980) Regional cerebral blood flow measurements by ${ }^{133}$ xenon-inhalation: Methodology and applications in neuropsychology and psychiatry. Brain and Language, 9, 9-34.

Rowe BM, Berkovic ST, Sia STB, et al. (1989) Localisation of epileptic foci with postictal single photon emission computerised tomography. Annals of Neurology, 26, 660-668.

Ryding E, Ingmar R, Elmqvist D and Ingvar DH (1988) SPECT measurements with ${ }^{99 \mathrm{~m}} \mathrm{HM}-\mathrm{PAO}$ in focal epilepsy. Journal of Cerebral Blood Flow Metabolism, 8 (Suppl.), S95-S100.

Ryding E, Sjoholm H, Skeidsvoll H and Elmqvist D (1989) Delayed increase in hemispheric cerebral blood flow during Wada test demonstrated by $99 \mathrm{mTc}$-HMPAO single photon emission computer tomography. Acta Neurologica Scandinavica, 80, 248-254.

Scoville WB and Milner B (1957) Loss of recent memory after bilateral hippocampal lesions. Journal of Neurology, Neurosurgery and Psychiatry, 20, 11-21.

Shedlack KJ, Hunter R, Wyper D, et al. (1991) The pattern of cerebral activity underlying verbal fluency shown by split-dose single photon emission computerised tomography (SPET or SPECT) in normal volunteers. Psychological Medicine, 21, 687-696.

Smith ML (1989) Memory disorders with temporal lobe lesions. In: Handbook of Neuropsychology, Vol. 3 (Eds F Boller and J Grafman). Elsevier Science Publishers, Amsterdam.

Squire LR, Ojemann JG, Miezin FM, et al. (1992) Activation of the hippocampus in normal humans: A functional anatomical study of memory. Proceedings of the National Academy of Sciences, 89, 1837-1841.

Stoddard HF (1987) Design and performance of the Cleon twodimensional, focused ray geometry scanner for single photon tomography. In: Physics and Engineering of Medical Engineering (Ed. J Guzzardi). Martinus Nijhoff, New Jersey.

Todd-Pokropek A (1984) Which SPECT? Nuclear Medicine Communications, 5, 421-437.

Wada J (1949) A new method for the determination of the side of cerebral speech dominance. A preliminary report on the intracarotid injection of sodium amytal in man. Medicine and Biology, 14, 221-222.

Wada J, Clark R and Hamm A (1975) Cerebral hemispheric asymmetry in humans. Archives of Neurology, 32, 239-245.

(Received 30 July 1993; accepted as revised 14 September 1993) 


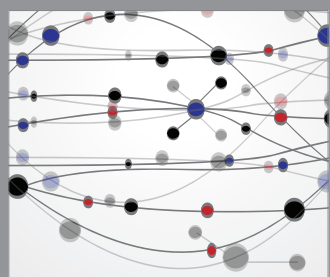

The Scientific World Journal
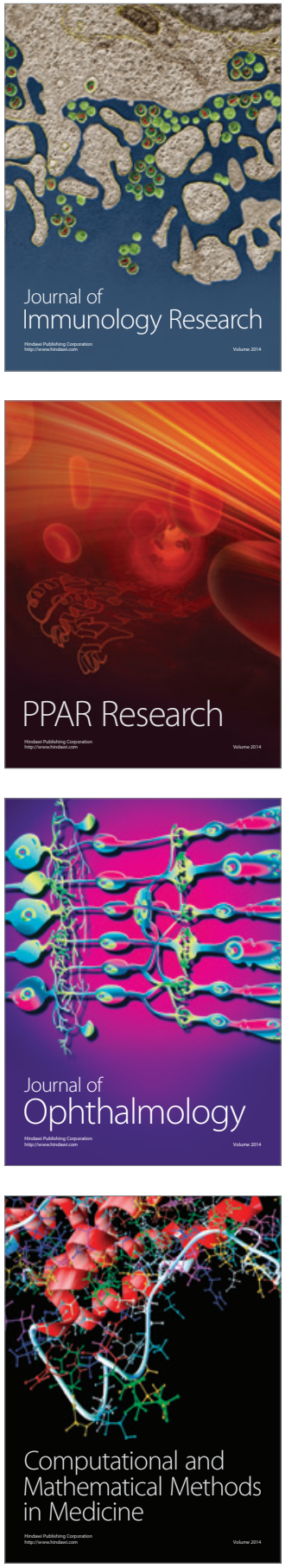

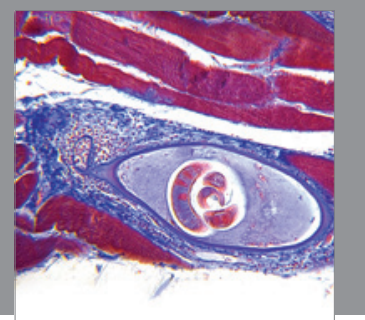

Gastroenterology

Research and Practice
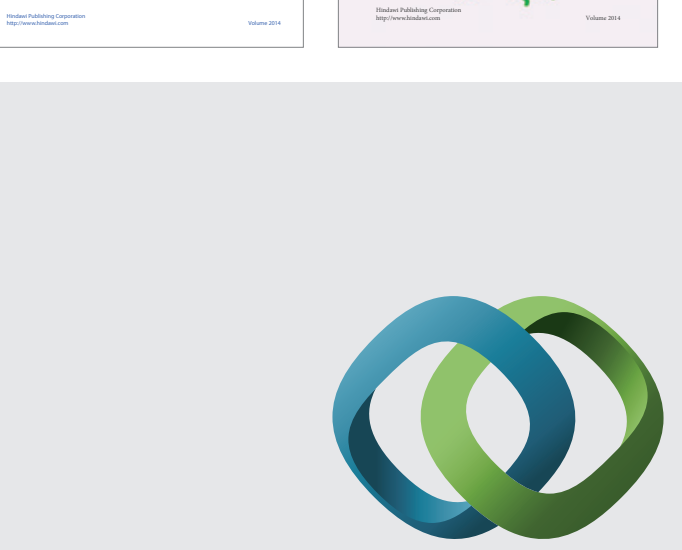

\section{Hindawi}

Submit your manuscripts at

http://www.hindawi.com
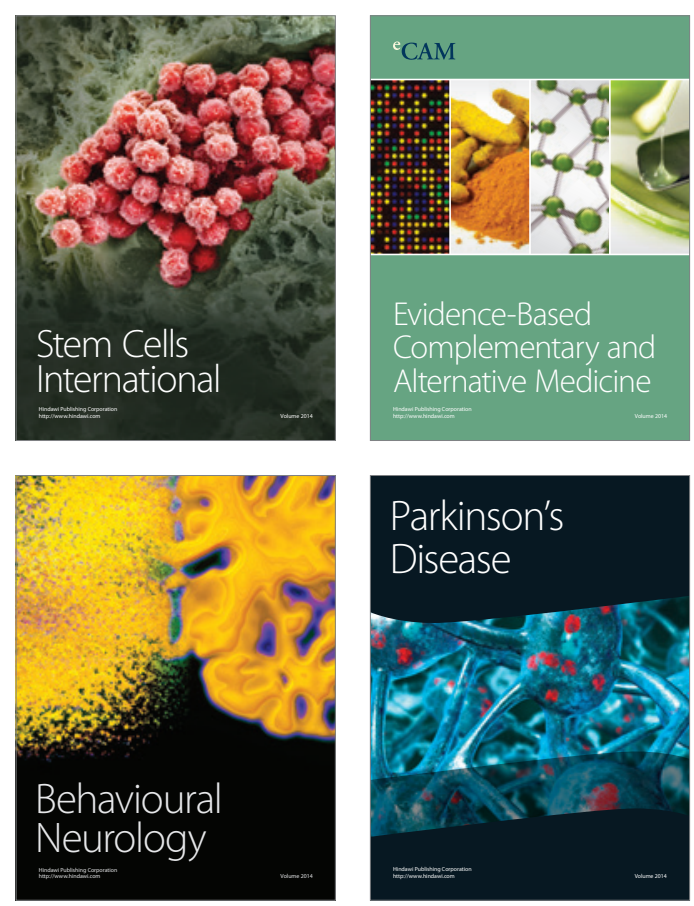

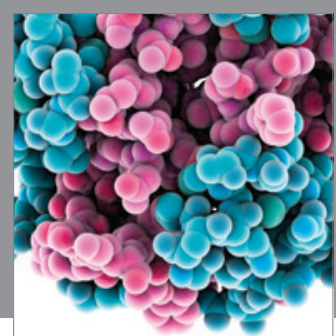

Journal of
Diabetes Research

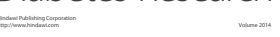

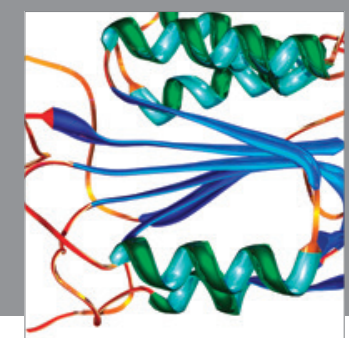

Disease Markers
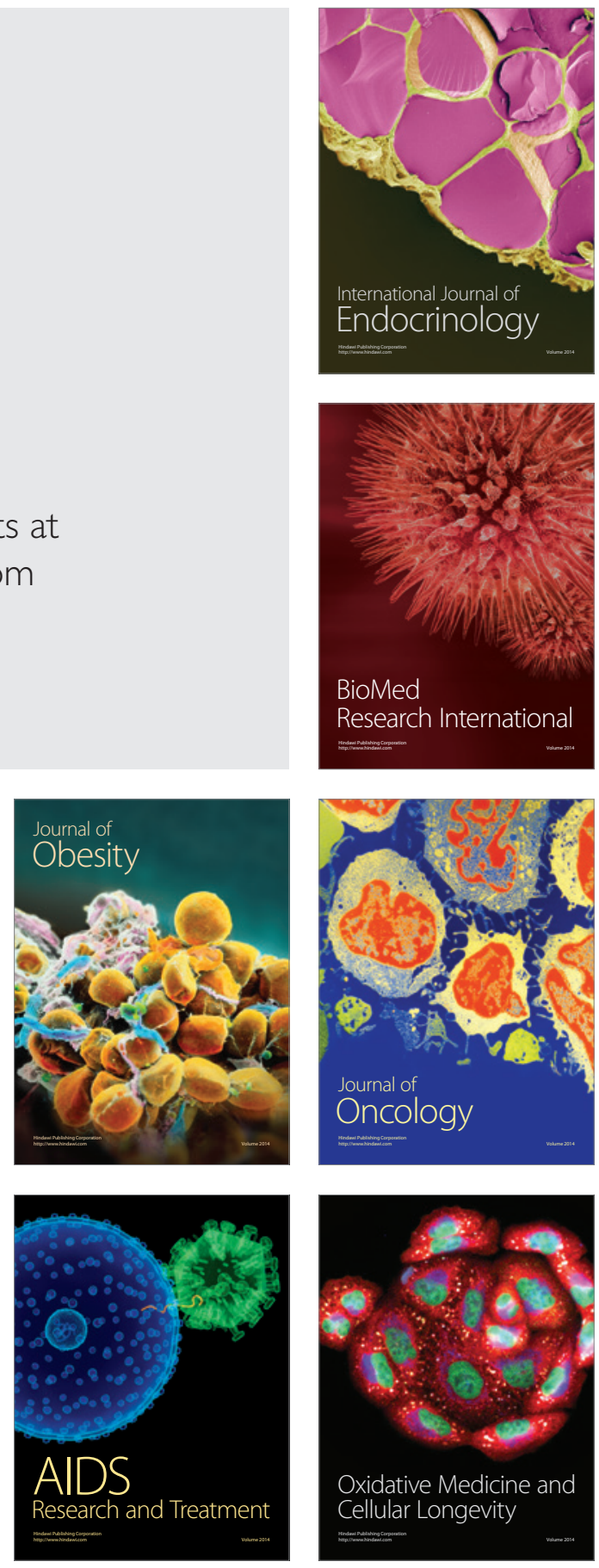